\title{
Efficacy of therapeutic plasma exchange on angiotensin II type-1 receptor antibodies on two kidney transplant recipients
}

\author{
Chisa Yamada ${ }^{1}$ () | Yihung Huang ${ }^{2}$ | Silas Norman ${ }^{3}$ | Abhijit Naik ${ }^{3}$ | Omar Moussa ${ }^{4}$ | \\ Milagros Samaniego ${ }^{5}$ । Laura Cooling ${ }^{1}$ (i)
}

${ }^{1}$ Division of Transfusion Medicine, Department of Pathology, University of Michigan, Ann Arbor, Michigan

${ }^{2}$ Section of Transplant Nephrology, Department of Internal Medicine, University of California, Davis, Davis, California

${ }^{3}$ Division of Nephrology, Department of Internal Medicine, University of Michigan, Ann Arbor, Michigan

${ }^{4}$ Division of Histocompatibility Laboratory, Department of Pathology, University of Michigan, Ann Arbor, Michigan

${ }^{5}$ Department of Medicine, Henry Ford Transplant Institute, Detroit, Michigan

Correspondence

Chisa Yamada, M.D., Blood Bank/Transfusion Medicine, Department of Pathology, University of Michigan, 1500 E. Medical Center Dr. SPC 5054, 2F228 UH, Ann Arbor, MI 48109-5054.

Email: yamadac@med.umich.edu

\begin{abstract}
Background: Angiotensin II type-1 receptor antibody (AT1RAb) has been reported to cause antibody mediated rejection (AMR) in kidney transplant recipients possibly by contraction of renal arteries. We here report 2 kidney transplant recipients with elevated AT1RAbs and negative HLA donor specific antibodies (DSA) and antimajor histocompatibility complex class I chain-related gene A (MICA) Abs who received therapeutic plasma exchange (TPE) treatment followed by IVIG.

Case 1: Thirty-eight-year-old patient received second kidney transplant for end stage renal disease (ESRD) with chronic rejection. Three years post-transplant, she developed AMR with AT1RAb level $>40 \mathrm{U} / \mathrm{mL}$. She received 5 TPE and AT1RAb decreased by $20 \%$, and biopsy showed improvement of AMR. She received another 3 TPE and AT1RAb decreased by $60 \%$. Her creatinine $(\mathrm{Cr})$ was stabilized at around $1.4 \mathrm{mg} / \mathrm{dL}$.

Case 2: Twenty-four-year-old patient received kidney transplant for ESRD with unclear etiology. Two weeks post-transplant, her Cr rose with AT1RAb level at $18 \mathrm{U} / \mathrm{mL}$ and biopsy showed possible AMR. She received 6 TPE treatments and AT1RAb decreased by 55\% and biopsy showed improvement of AMR. She received weekly TPE for subsequently rising AT1RAb but TPE was discontinued because of unsuccessful decrease of AT1RAb. Her Cr was stabilized at around $1.7 \mathrm{~mL} / \mathrm{dL}$.

Conclusion: We reported 2 patients who received TPE treatments to decrease AT1RAbs. A course of TPE treatment successfully decreased AT1RAb. Histological improvement was observed quickly and $\mathrm{Cr}$ was also stabilized following the TPE treatment. Further study is necessary to determine the optimal use of TPE in renal transplant recipients with AT1RAbs.
\end{abstract}

\section{KEYWORDS}

angiotensin II type-1 receptor antibody, antibody mediated rejection, kidney transplantation, therapeutic plasma exchange

\section{1 | INTRODUCTION}

Some renal transplant recipients develop antibody mediated rejection (AMR) without detectable HLA donor specific antibodies (DSA) in sera. In recent years, angiotensin II type-1

Institution at which the work was performed: University of Michigan. receptor antibody (AT1RAb) has been reported to be associated with kidney AMR, especially refractory AMR, possibly by contraction of renal arteries. Therapeutic plasma exchange (TPE) has been increasingly applied to reduce HLA DSA in kidney AMR; less is known regarding the role of TPE in AMR because of non-HLA Abs such as AT1RAb. Although there are several published reports describing the use of TPE 
in the treatment of AT1RAb associated AMR, the efficacy of TPE in reducing AT1RAb levels has not been reported.

Our institution has been implementing AT1RAb testing for the kidney transplant recipients with biopsy proven or suspicious for AMR on renal biopsy without HLA DSA, and a course of treatment with 5-6 TPE followed by IVIG has been applied on those patients as same as on patients with HLA DSA. We investigated AT1RAb and creatinine (Cr) levels before and after TPE treatments in those patients and report 2 patients here.

\section{2 | MATERIALS AND METHODS}

\section{1 | Patients}

Renal transplant recipients with elevated AT1RAbs, negative HLA DSA and negative antimajor histocompatibility complex class I chain-related gene A (MICA) Abs who received TPE treatments for histologically proven or suspicious for AMR between January 2014 and December 2016 were identified in our institutional renal transplant database. Among those patients, a total of 9 patients with elevated AT1RAb levels before TPE treatment were identified. Among them, 2 patients had positive HLA DSA, 3 patients lack follow-up laboratory tests, and 2 patients had follow-up AT1RAb tests more than 3 months after the last TPE procedure. The remaining 2 patients' demographics, causes of kidney failure, types of donor, graft age at the first TPE procedure, and angiotensin II receptor blockers (ARB) use are shown in Table 1 . Of note, no patient received rituximab at the time of TPE treatment.

\section{2 | Angiotensin II type-1 receptor antibody}

Serum AT1RAb level was measured using enzyme-linked immunosorbent assay (ELISA) test (One Lambda, Canoga Park, CA) and reported as high $(>17 \mathrm{U} / \mathrm{mL})$, intermediate $(12-17 \mathrm{U} / \mathrm{mL})$, and negative $(<12 \mathrm{U} / \mathrm{mL})$. Because of the lack of protocol to test AT1RAb level, AT1RAb levels were tested in various time points after TPE treatment. No patient was tested for AT1RAb prior to their transplantations and this test is currently unavailable for validation process at our institution.

\section{3 | Therapeutic apheresis}

In our facility, renal transplant recipients with high DSA against HLAs or non-HLA Abs and/or biopsy proven AMR receive a course of TPE treatment (5-6 TPE followed by IVIG infusion each time). Additional TPE may be performed after the initial course based on the patient's response to the treatment or post-TPE antibody level (eg, AT1RAb, HLA DSA). TPE procedures were performed using either Cobe Spectra or Spectra Optia Apheresis System (TerumoBCT, Lakewood, CO), and one plasma volume was exchanged per procedure. Citrate dextrose solution A was used as an anticoagulant and 5\% albumin was used as a replacement fluid.

\section{3 | RESULTS}

\section{1 | Case 1}

Patient A was a 42-year-old female with focal segmental glomerulosclerosis who received her first living related donor kidney transplant at age 22, and a second deceased donor transplant because of a chronic rejection of the transplanted kidney at age 38. Three years post-transplant, her $\mathrm{Cr}$ started to rise from 0.7 to $1.35 \mathrm{mg} / \mathrm{dL}$ and a biopsy showed Banff grade $2 \mathrm{AMR}^{1,2}$, grade $2 \mathrm{~A}$ T-cell mediated rejection (TCMR), and grade 3 interstitial fibrosis and tubular atrophy (IFTA) (Table 2). Serum HLA DSA and antiMICA Abs were negative, but high level AT1RAb was identified at $>40 \mathrm{U} / \mathrm{mL}$ (Figure 1, A). She received 5 TPEs each followed by IVIG every other day over 9 days and started ARB treatment. The AT1RAb level measured 2 days after a course of TPE treatment decreased to $32 \mathrm{U} / \mathrm{mL}(20.0 \%$ reduction), and histology showed grade $0 \mathrm{AMR}$ and $1 \mathrm{~B}$ TCMR 26 days after the last TPE treatment. Over the course of the next month, her $\mathrm{Cr}$ slowly increased to $1.64 \mathrm{mg} / \mathrm{dL}$, accompanied by a rise in AT1RAb $(>40 \mathrm{U} / \mathrm{mL})$. She was treated with 3 more TPE treatments every other day and her AT1RAb level decreased to $16 \mathrm{U} / \mathrm{mL}$ (60.0\% reduction). Although AT1RAb level increased slightly to $20 \mathrm{U} / \mathrm{mL}$, kidney biopsy still showed grade 0 AMR 3 months after the last TPE. Her $\mathrm{Cr}$ at approximately 1 year after the last TPE was at around $1.4 \mathrm{mg} / \mathrm{dL}$, and her most recent $\mathrm{Cr}$ in January 2018 was $1.31 \mathrm{mg} / \mathrm{dL}$. HLA DSA has been negative to date.

TABLE 1 Demographics and angiotensin II antagonist use

\begin{tabular}{|c|c|c|c|c|c|c|c|c|}
\hline Patient & Gender & Race & $\begin{array}{l}\text { Cause of kidney } \\
\text { failure }\end{array}$ & $\begin{array}{l}\text { Type of } \\
\text { donor }\end{array}$ & $\begin{array}{l}\text { Age at } \\
\text { transplant }\end{array}$ & $\begin{array}{l}\text { Age at } \\
\text { first TPE }\end{array}$ & $\begin{array}{l}\text { Graft age at first } \\
\text { TPE, days }\end{array}$ & $\begin{array}{l}\text { ARB } \\
\text { use }\end{array}$ \\
\hline \multirow[t]{2}{*}{ A } & Female & AA & FSGS & Living related & 22 & - & - & - \\
\hline & & & Chronic rejection & Deceased & 38 & 42 & 1098 & Yes \\
\hline B & Female & $\mathrm{AA}$ & Unclear etiology & Deceased & 24 & 25 & 61 & No \\
\hline
\end{tabular}

Abbreviation: AA, African American; ARB, angiotensin II receptor blocker; DM, diabetes mellitus; FSGS, focal segmental glomerulosclerosis; HTN, hypertension; TPE, therapeutic plasma exchange. 
TABLE 2 Kidney biopsy results

\begin{tabular}{|c|c|c|c|c|c|c|}
\hline Patient & $\begin{array}{l}\text { Renal } \\
\text { biopsy date }\end{array}$ & $\begin{array}{l}\text { AMR } \\
\text { grade }\end{array}$ & $\begin{array}{l}\text { TCMR } \\
\text { grade }\end{array}$ & $\begin{array}{l}\text { IFTA } \\
\text { grade }\end{array}$ & $\begin{array}{l}\text { Peritubular } \\
\text { capillary } \mathbf{C 4 d}\end{array}$ & Change regarding AMR \\
\hline \multirow[t]{3}{*}{ A } & July 10,2015 & 2 & $2 \mathrm{~A}$ & 3 & Focal, weak & Severe tubulitis and capillaritis \\
\hline & August 14, 2015 & 0 & $1 \mathrm{~B}$ & 3 & Negative & $\begin{array}{l}\text { Reduction in interstitial inflammation, improvement in the } \\
\text { capillaritis, persistent tubulitis }\end{array}$ \\
\hline & $\begin{array}{l}\text { November } \\
20,2015\end{array}$ & 0 & - & 3 & Negative & No change compared to previous biopsy \\
\hline \multirow[t]{3}{*}{ B } & March 18, 2016 & 0 & 0 & 1 & Negative & Segmental glomerulitis and vasculopathy \\
\hline & April 28, 2016 & 0 & 0 & 2 & Negative & Increased interstitial fibrosis \\
\hline & July 5, 2016 & 0 & 0 & 1 & Negative & Focal glomerulopathy and interstitial fibrosis \\
\hline
\end{tabular}

Abbreviation: AMR, antibody mediated rejection; ICMR, T-cell mediated rejection; IFTA, interstitial fibrosis and tubular atrophy; TPE, therapeutic plasma exchange.

(A)
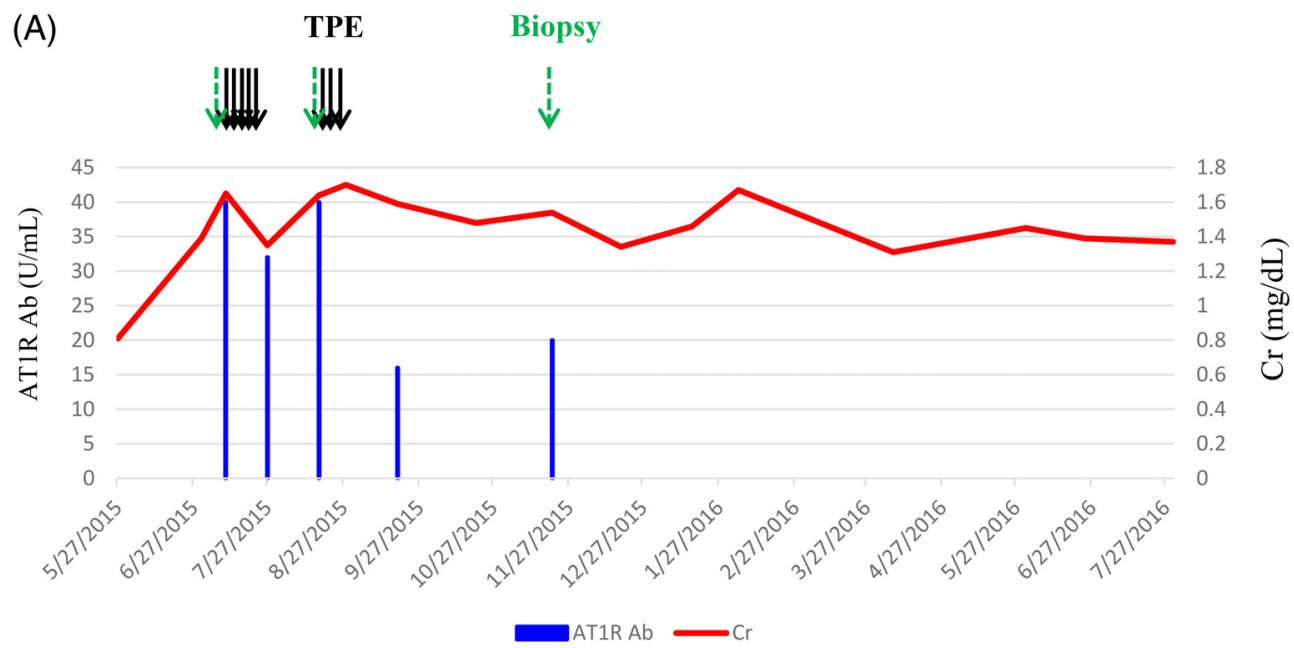

(B)

TPE Biopsy
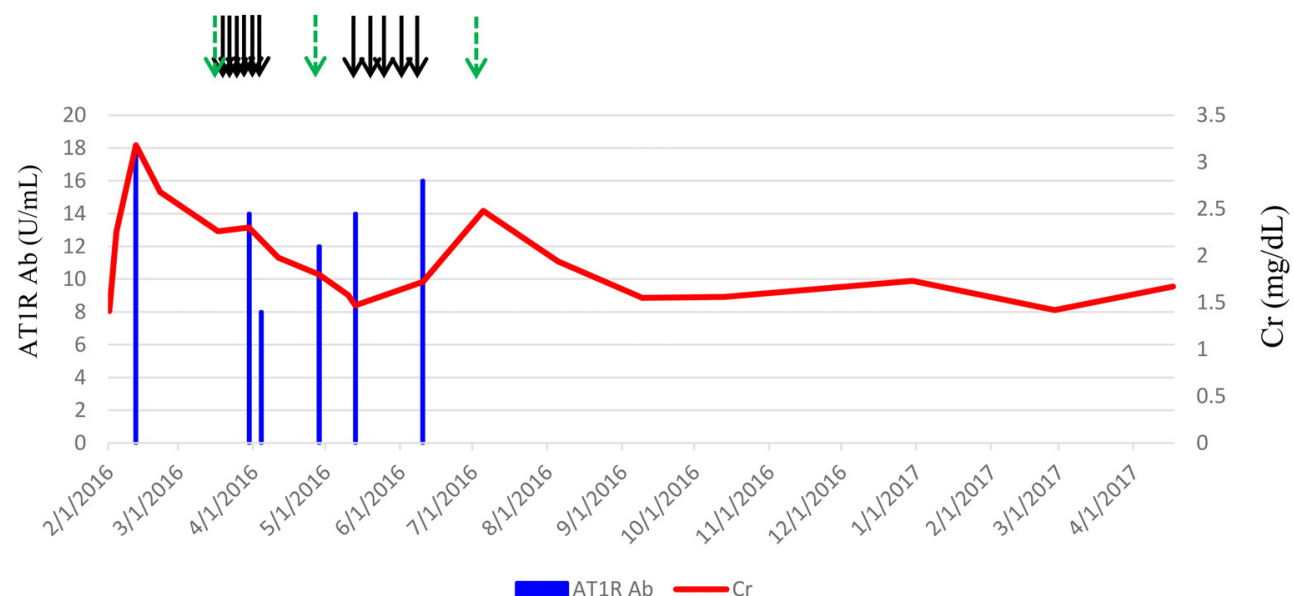

FIGURE 1 AT1RAb level, creatinine level and the timing of TPE and kidney biopsies. Abbreviation: AT1RAb: angiotensin II type-1 antibody; Cr, creatinine; TPE, therapeutic plasma exchange

\section{2 | Case 2}

Patient B was a 25-year-old female with renal failure because of unclear etiology who received a deceased donor kidney transplant at age 24 . Her Cr started to rise 2 weeks post-transplant from 1.40 to $2.68 \mathrm{mg} / \mathrm{dL}$ (Figure 1,B) without detectable HLA DSA and anti-MICA Abs. A biopsy showed Banff grade 0 AMR, grade 0 TCMR and grade 1 IFTA (Table 2), but had focally severe endothelial swelling and cell injury suggesting process for AMR. Her AT1RAb level was found to be elevated at $18 \mathrm{U} / \mathrm{mL}$. She received 6 TPE each followed by IVIG every other day over 11 days and her AT1RAb level decreased to $8 \mathrm{U} / \mathrm{mL}(55.6 \%$ reduction). Her $\mathrm{Cr}$ was improved to $1.98 \mathrm{mg} / \mathrm{dL}$, and biopsy also showed improved arteriopathy 24 days after a course of TPE. However, she was started on weekly TPE treatment for the next 5 weeks because of rising AT1RAb $(12 \mathrm{U} / \mathrm{mL})$ 
2 weeks after completing TPE. Her AT1RAb levels measured before her fifth weekly TPE treatment showed increase to $16 \mathrm{U} / \mathrm{mL}$, therefore, TPE was discontinued. Kidney biopsy 25 days after the last TPE still showed grade 0 AMR. Clinically, her $\mathrm{Cr}$ remained stable at around $1.7 \mathrm{~mL} / \mathrm{dL}$ for 10 months after the last TPE. She had subsequent CMV infection, which caused mild deterioration of kidney function and her most recent $\mathrm{Cr}$ in March 2018 was $2.75 \mathrm{mg} / \mathrm{dL}$. HLA DSA has been negative to date.

\section{4 | DISCUSSION}

The majority of AMR in renal transplantation is attributed to DSA to HLA antigens. In recent years, however, several nonHLA Abs have been linked to AMR including anti-endothelial cell Abs, MICA Abs, antiAT1RAbs, anti-endothelin-1 type A receptor Abs, anti-poly-reactive Abs, anti-LG3 Abs and anti-vimentin Abs. ${ }^{3,4}$

AT1R mediates physiological actions of angiotensin II (ATII) in many organs including kidney. ATII promotes vasoconstriction, inflammation, thrombosis, and vascular remodeling, ${ }^{5}$ and AT1R regulates arterial blood pressure, electrolyte and water balance, and renal function. ${ }^{6}$ Therefore, hyperactivation of AT1R by AT1RAbs can cause hypertension and kidney dysfunction with vasoconstriction and thrombosis. AT1RAb can be autoimmune or alloimmune $\mathrm{Ab}$. Preoperative positive AT1R using ELISA is found in $23 \%-47.2 \%$ of the patients who received first or multiple kidney transplantations, ${ }^{7-10}$ de novo development of AT1RAb after the transplantation is reported in $45 \%$ of the pediatric patients, ${ }^{10}$ and $9.4 \%$ of the patients with rejection are reported to have AT1RAbs. ${ }^{11}$ AT1RAb is also associated with steroid refractory vascular rejection or higher rate of late graft loss. ${ }^{7,8,10,12-14}$ Banasik et al. ${ }^{8}$ reported that patients with AT1RAbs run a higher risk of graft failure independently of classical immunological risk factors, but not acute rejection or hypertension.

There are some reports in which TPE was used to treat AMR in kidney transplant recipients with AT1RAbs. Jobert et al. reported a case of AMR in a kidney transplant recipient with AT1RAb, who received 6 TPE procedures with 1.5 plasma volume exchange. ${ }^{15}$ Fuss et al. used 3-11 TPE procedure to treat 6 kidney AMR patients with AT1RAbs. ${ }^{16}$ Lee et al. ${ }^{17}$ treated 11 cases of AT1RAb associated AMR with a combination of TPE and IVIG, sometimes followed by rituximab (9/12), antithymocyte globulin (5/12), or bortezomib (1/12). All patients experienced clinical improvement and/or resolution of AMR. None of these reports, however, measured AT1RAb level after TPE treatment or analyzed role and efficacy of TPE in reducing AT1RAb, and correlation with clinical outcomes. Our cases show that 1 course of 5-6 TPE treatment, followed by IVIG, successfully reduces AT1RAb level by $20.0 \%$ and $55.6 \%$, median $37.8 \%$. This rate is similar to the reduction rates of HLA DSA in kidney transplant recipients with AMR that we previously reported elsewhere. ${ }^{18}$ However, the effect of TPE was temporally and AT1RAb rebound and increase over the time were also observed, which indicates that follow-up of these patients is critical. Some of the cases in these reports received a combination of treatment with TPE and ARB, however, report for an effect of ARB only on renal AMR caused by AT1RAbs was not found at this time. It may be because transplant nephrologists have been reluctant to use ARB because of possible decreases in organ perfusion and filtration mentioned by Dragun et al. ${ }^{13}$

The improvement of kidney function was also observed after the TPE treatment. Cr was stabilized without further increase. Therefore, it may be important to treat patients with biopsy proven or suspicious for AMR without HLA DSA, even if AT1RAb testing is not available, to prevent further deterioration of kidney function, especially when those patients were proven to have AT1RAbs which are thought to have a higher probability for late graft failure as mentioned above. In addition, there is a possibility that these patients had other unknown antibodies against donor kidney in addition to AT1RAb that could be also decreased by TPE treatment and medications, which might be taking some role in improvement of biopsy or kidney function.

The current Banff histologic classification for kidney AMR is based primarily on AMR resulting from HLA DSA. ${ }^{1,2}$ Although the Banff classification is also used for non-HLA AMR, the mechanisms underlying renal damage and dysfunction may differ, which could impact biopsy results. Specifically, HLA Abs frequently cause tissue damage through complement cascade activation. As a result, biopsy results in HLA DSA-mediated AMR often show C4d positivity on peritubular capillaries. In contrast, kidney failure and AMR because of AT1RAb are thought to be caused by renal artery vasoconstriction. ${ }^{19}$ The reason that a negative $\mathrm{C} 4 \mathrm{~d}$ is a common finding in AT1RAb-mediated $\mathrm{AMR}^{15,16,20,21}$ may be because complement may not be involved for kidney dysfunction. In addition, vasculitis, thrombosis, and glomerulopathy may be common and the predominant histologic findings. Lim et al. ${ }^{22}$ did not find an association between peritubular capillary $\mathrm{C} 4 \mathrm{~d}$ staining and AT1RAb, however, reported several cases with positive C4d staining. In our 2 patients, patient $\mathrm{A}$ had a weak $\mathrm{C} 4 \mathrm{~d}$ staining which disappeared after TPE treatment and patient B had negative $\mathrm{C} 4 \mathrm{~d}$ staining on biopsies. Overall, the histological abnormalities were improved after a course of TPE treatment in both patients, which may suggest removing AT1RAbs by TPE and suppression of Ab production by medications, may be able to decrease vasoconstriction quickly and to provide prevention of further damage on kidney.

In summary, we presented a first report on efficacy of TPE treatment followed by IVIG in reducing AT1RAb level in 2 patients. A course of TPE decreased AT1RAb by median $37.8 \%$ measured within 1 month after the last TPE. 
However, rebound and subsequent increase of AT1RAb was also observed, therefore, follow-up of AT1RAb level is important when AT1RAb lab test is available. Histological improvement in AMR was observed after a course of TPE treatment and $\mathrm{Cr}$ did not show further deterioration of the kidney function in several months, which may suggest decreased level of AT1RAb by treatment with TPE followed by IVIG may be able to decrease vasoconstriction caused by AT1RAb and to prevent further deterioration of kidney function. A further study is needed to determine if TPE treatment is more favorable over ARB treatment, and optimal use of TPE in renal transplant patients with AT1RAbs.

\section{ORCID}

Chisa Yamada (DD https://orcid.org/0000-0002-1968-2385 Laura Cooling (DD https://orcid.org/0000-0003-0216-1599

\section{REFERENCES}

1. Loupy A, Haas M, Solez K, et al. The Banff 2015 Kidney Meeting Report: current challenges in rejection classification and prospects for adopting molecular pathology. Am J Transplant. 2017;17:28-41.

2. Haas M, Loupy A, Lefaucheur C, et al. The Banff 2017 Kidney Meeting Report: revised diagnostic criteria for chronic active $\mathrm{T}$ cell-mediated rejection, antibody-mediated rejection, and prospects for integrative endpoints for next-generation clinical trials. Am J Transplant. 2018;18(2):293-307.

3. Delville M, Charreau B, Rabant M, Legendre C, Anglicheau D. Pathogenesis of non-HLA antibodies in solid organ transplantation: where do we stand? Hum Immunol. 2016;77:1055-1062.

4. Michielsen LA, van Zuilen AD, Krebber MM, Verhaar MC, Otten HG. Clinical value of non-HLA antibodies in kidney transplantation: still an enigma? Transplant Rev. 2016;30:195-202.

5. Dzau VJ. Theodore Cooper lecture: tissue angiotensin and pathobiology of vascular disease: a unifying hypothesis. Hypertension. 2001;37:1047-1052.

6. de Gasparo M, Catt KJ, Inagami T, Wright JW, Unger T. International union of pharmacology. XXIII. The angiotensin II receptors. Pharmacol Rev. 2000;52:415-472.

7. Giral M, Foucher Y, Dufay A, et al. Pretransplant sensitization against angiotensin II type 1 receptor is a risk factor for acute rejection and graft loss. Am J Transplant. 2013;13:2567-2576.

8. Banasik M, Boratynska M, Koscielska-Kasprzak K, et al. The influence of non-HLA antibodies directed against angiotensin II type 1 receptor (AT1R) on early renal transplant outcomes. Transpl Int. 2014;27:1029-1038.

9. Deltombe C, Gillaizeau F, Anglicheau D, et al. Is pre-transplant sensitization against angiotensin II type 1 receptor still a risk factor of graft and patient outcome in kidney transplantation in the anti-HLA Luminex era? A retrospective study. Transpl Int. 2017;30:1150-1160.
10. Pearl MH, Zhang Q, Palma Diaz MF, et al. Angiotensin II type 1 receptor antibodies are associated with inflammatory cytokines and poor clinical outcomes in pediatric kidney transplantation. Kidney Int. 2018;93(1):260-269.

11. Lee H, Kim JI, Moon IS, et al. Investigation of serum angiotensin II type 1 receptor antibodies at the time of renal allograft rejection. Ann Lab Med. 2015;35:314-320.

12. Dragun D, Brasen JH, Schonemann C, et al. Patients with steroid refractory acute vascular rejection develop agonistic antibodies targeting angiotensin II type 1 receptor. Transplant Proc. 2003;35:2104-2105.

13. Dragun D, Muller DN, Brasen JH, et al. Angiotensin II type 1-receptor activating antibodies in renal-allograft rejection. $N$ Engl J Med. 2005;352: 558-569.

14. Taniguchi M, Rebellato LM, Cai J, et al. Higher risk of kidney graft failure in the presence of anti-angiotensin II type-1 receptor antibodies. Am J Transplant. 2013;13:2577-2589.

15. Jobert A, Rao N, Deayton S, et al. Angiotensin II type 1 receptor antibody precipitating acute vascular rejection in kidney transplantation. Nephrology. 2015;20(Suppl 1):10-12.

16. Fuss A, Hope CM, Deayton $S$, et al. C4d-negative antibody-mediated rejection with high anti-angiotensin II type I receptor antibodies in absence of donor-specific antibodies. Nephrology. 2015;20:467-473.

17. Lee J, Park Y, Kim BS, et al. Clinical implications of angiotensin II type 1 receptor antibodies in antibody-mediated rejection without detectable donor-specific HLA antibodies after renal transplantation. Transplant Proc. 2015;47:649-652.

18. Yamada C, Ramon DS, Cascalho $M$, et al. Efficacy of plasmapheresis on donor-specific antibody reduction by HLA specificity in post-kidney transplant recipients. Transfusion. 2015;55:727-735. quiz 6.

19. Lukitsch I, Kehr J, Chaykovska L, et al. Renal ischemia and transplantation predispose to vascular constriction mediated by angiotensin II type 1 receptor-activating antibodies. Transplantation. 2012;94:8-13.

20. Reinsmoen NL, Lai $\mathrm{CH}$, Heidecke $\mathrm{H}$, et al. Anti-angiotensin type 1 receptor antibodies associated with antibody mediated rejection in donor HLA antibody negative patients. Transplantation. 2010;90:1473-1477.

21. Pearl MH, Leuchter RK, Reed EF, Zhang Q, Ettenger RB, Tsai EW. Accelerated rejection, thrombosis, and graft failure with angiotensin II type 1 receptor antibodies. Pediatr Nephrol. 2015;30:1371-1374.

22. Lim MA, Palmer M, Trofe-Clark J, et al. Histopathologic changes in anti-angiotensin II type 1 receptor antibody-positive kidney transplant recipients with acute rejection and no donor specific HLA antibodies. Нum Immunol. 2017;78:350-356.

How to cite this article: Yamada C, Huang Y, Norman S, et al. Efficacy of therapeutic plasma exchange on angiotensin II type-1 receptor antibodies on two kidney transplant recipients. J Clin Apher. 2018;33:673-677. https://doi.org/10.1002/jca.21657 